\title{
Parents' Perspectives and Clinical Effectiveness of Cranial-Molding Orthoses in Infants With Plagiocephaly
}

\author{
Hyo Sun Lee, MD, Sang Jun Kim, MD, PhD, Jeong-Yi Kwon, MD, PhD \\ Department of Physical and Rehabilitation Medicine, Samsung Medical Center, \\ Sungkyunkwan University School of Medicine, Seoul, Korea
}

Objective To investigate the clinical effectiveness of and parents' perspectives on cranial-molding orthotic treatment.

Methods Medical charts were reviewed for 82 infants treated for plagiocephaly with cranial-molding orthoses in our clinic from April 2012 to July 2016 retrospectively. Infants who were clinically diagnosed with positional plagiocephaly and had a Cranial Vault Asymmetry Index (CVAI) of more than 3.5\% were included. Pre- and post-treatment CVAI was obtained by three-dimensional head-surface laser scan. Parents' perceptions of good outcome (satisfaction) were evaluated with the Goal Attainment Scale (GAS). The GAS score assessed how much the parent felt that his or her initial goal for correcting the skull asymmetry was achieved after the treatment.

Results The compliance with cranial-molding orthoses was $90.2 \%$ (74 of 82 infants). There were 53 infants (65\% of the 82 infants) who had adverse events with the cranial-molding orthoses during the study. Heat rash was found in 29 cases $(35.4 \%)$ and was the most common adverse event. The mean GAS T-score was $51.9 \pm 10.2$. A GAS T-score of 0 or more was identified for $71.6 \%$ of parents. The GAS T-score was significantly related to the age $(\mathrm{p}<0.001)$, the initial CVAI, and the difference of CVAI during the treatment $(\mathrm{p}<0.001)$.

Conclusion Parents' perception of good outcome was correlated with the anthropometric improvement in cranialmolding orthotic treatment in infants with plagiocephaly. A high percentage of parents felt that the treatment met their initial goals in spite of a high occurrence of adverse events.

Keywords Orthotic devices, Plagiocephaly, Treatment outcome, Cephalymetry

Received January 12, 2018; Accepted March 15, 2018

Corresponding author: Jeong-Yi Kwon

Department of Physical and Rehabilitation Medicine, Samsung Medical Center, Sungkyunkwan University School of Medicine, 81 Irwon-ro, Gangnamgu, Seoul 06351, Korea. Tel: +82-2-3410-2984, Fax: +82-2-3410-2820, E-mail: jeongyi.kwon@samsung.com

ORCID: Hyo Sun Lee (http://orcid.org/0000-0002-5933-8642); Sang Jun Kim (http://orcid.org/0000-0002-0479-7526); Jeong-Yi Kwon (http://orcid. org/0000-0001-7537-3176).

(c) This is an open-access article distributed under the terms of the Creative Commons Attribution Non-Commercial License (http://creativecommons.org/ licenses/by-nc/4.0) which permits unrestricted noncommercial use, distribution, and reproduction in any medium, provided the original work is properly cited. Copyright $\odot 2018$ by Korean Academy of Rehabilitation Medicine 


\section{INTRODUCTION}

Plagiocephaly, derived from the Greek words 'plagio', meaning 'oblique', and 'kephale', which means 'head', describes an asymmetric deformity of the skull that can occur anteriorly or posteriorly [1]. Deformational plagiocephaly arises from repeated external pressure to the same area of the flexible cranium during the fetal or neonatal periods [2]. When this repeated pressure is applied to one side of the occiput, it results in ipsilateral occipital flattening, frontal bone protrusion, and anterior shifting of the ipsilateral ear [3]. Deformational plagiocephaly and brachycephaly affect approximately $20 \%$ of infants [4]. This reflects an increase in the incidence of the condition after several countries implemented public-health campaigns that encouraged parents to position their babies in a supine sleeping position in order to prevent sudden infant death syndrome [5].

In infants who were diagnosed with deformational plagiocephaly before the age of 6 months, deformational plagiocephaly was observed in nearly one-third of children re-examined at ages 2 to 3 years [6]. Treatments of plagiocephaly include repositioning, physical therapy, and cranial-molding orthoses [7]. Repositioning and physical therapy are routinely used prior to a trial of cranial-molding orthoses. Cranial-molding orthoses are generally custom-fitted orthoses that are designed to be worn 23 hours a day for several months, until either the child has achieved satisfactory cosmetic correction or has outgrown the helmet [8].

There has been debate among physicians about whether cranial-molding orthoses is more effective than repositioning or physical therapy. A prospective cohort study reported an equal effectiveness of cranial-molding orthoses and skull deformation following its natural course [9]. This study discouraged the use of cranial-molding orthoses as a standard treatment for infants with skull deformation. However, another prospective comparison study conducted for 36 posterior plagiocephaly patients reported a greater improvement in correction of asymmetry with cranial-molding orthoses than with repositioning [10]. A systematic review of cranial-molding orthoses for plagiocephaly demonstrated a more significant and faster improvement of cranial asymmetry in infants with deformational plagiocephaly, especially if the asymmetry was severe and the cranial-molding orthosis was applied early in infancy [8]. For infants who fail to improve with repositioning by age 6 months and who continue to have a severe deformity, many pediatric neurosurgeons and plastic surgeons recommend that these infants be treated with cranial-molding orthoses [7]. The Congress of Neurological Surgeons reported an evidence-based guideline for cranial-molding orthotic treatment in 2016. According to this guideline, Level II using cranial-molding orthoses is recommended when moderate to severe plagiocephaly is persistent after a course of conservative treatment, or when infants present moderate to severe plagiocephaly at an advanced age [8].

In childhood disease, evaluation of the parents' perspectives on various interventions is considered important in healthcare decision-making and evaluation [11]. Research on consumer perspectives, specifically the perspectives of the parents of children receiving the intervention, complements and extends data gathered from direct observation and provider perspectives on interventions, which can be more individualized and childcentered and/or family-centered based on understanding the parents' perspectives on therapies.

However, there is a lack of research on the parents' perspectives about cranial-molding orthoses for infants who were diagnosed with deformational plagiocephaly. Elwood et al. [12] reported that parents rated head-shape improvement in cranial-molding orthotic treatment as 4.06 out of 5 (range $3-5$, with 1 representing the least improvement and 5 representing the most). Katzel et al. [13] surveyed parent ratings of children's head shape before and after cranial-molding orthotic treatment and reported that parents perceived a large correction in head shape. The parents' ratings before and after cranialmolding orthotic treatment were 2.99 and 7.88 , respectively; a scale of 1 to 10 was used, with 1 representing abnormal and 10 representing normal.

However, there are no studies that have identified the factors that affect parental perspectives on cranial-molding orthoses. In this study, we assumed that the various clinical factors that were known to be related to the clinical outcomes of cranial-molding orthoses would affect the parents' perspectives on it. Therefore, the aim of this study was to investigate the parents' perspectives and the clinical effectiveness of cranial-molding orthoses in infants with plagiocephaly. 


\section{MATERIALS AND METHODS}

\section{Subjects}

Medical charts were reviewed for 82 infants treated for plagiocephaly by means of cranial-molding orthosis in our clinic from April 2012 to July 2016. This research was approved by the Institutional Review Board of Samsung Medical Center (IRB No. 2017-06-134-001). Infants who were clinically diagnosed with positional plagiocephaly and who had a Cranial Vault Asymmetry Index (CVAI) of more than $3.5 \%$ were included. The infants were clinically diagnosed as having positional plagiocephaly by a pediatric physiatrist when they had ipsilateral occipital flattening and the resulting frontal bone protrusion or asymmetric ear shifting. Of the 82 subjects, 8 were excluded in the final analysis because they had discontinued wearing the cranial-molding orthosis during the study period. Basic information, including the age at the initiation of cranial-molding orthotic treatment, accompanying disease, and past medical history was obtained.

\section{Anthropometric measurement}

Anthropometric measurements were obtained using a three-dimensional (3D) head-surface laser scan (Vorum, Vancouver, Canada). A cross-sectional plane through the sellion and both tragia was set as the reference plane and designated as the level 0 plane (Fig. 1A). The plane parallel to the level 0 plane that passed through the vertex of the head was designated as the level 10 plane. The portion of the cranium superior to the reference level 0 plane was divided into 9 equally spaced cross-sectional planes, each parallel to the reference plane. The following anthropometric measurements were obtained at levels 3 and 5 (Fig. 1) [14,15]: (1) the diagonal difference (mm), which is defined as the difference between the longer cranial diagonal and the shorter cranial diagonal, and (2) the CVAI (\%), which is defined as

$\frac{\text { long cranial diagonal }(\mathrm{mm}) \text {-short cranial diagonal }(\mathrm{mm})}{\text { short cranial diagonal }(\mathrm{mm})} \times 100$.

\section{Cranial-molding orthoses}

A cranial-molding orthosis (OrthoKorea, Seoul, Korea) was made for patients based on their initial 3D headsurface laser scan. It consisted of a polyethylene foam liner and a copolymer outer shell. We explained adverse events and precautions to parents when prescribing the cranial-molding orthoses and recommended that patients wear the cranial-molding orthoses for no more than 23 hours a day; 74 patients wore the cranialmolding orthoses until they reached the objective treatment goal or until the orthoses no longer fit their head

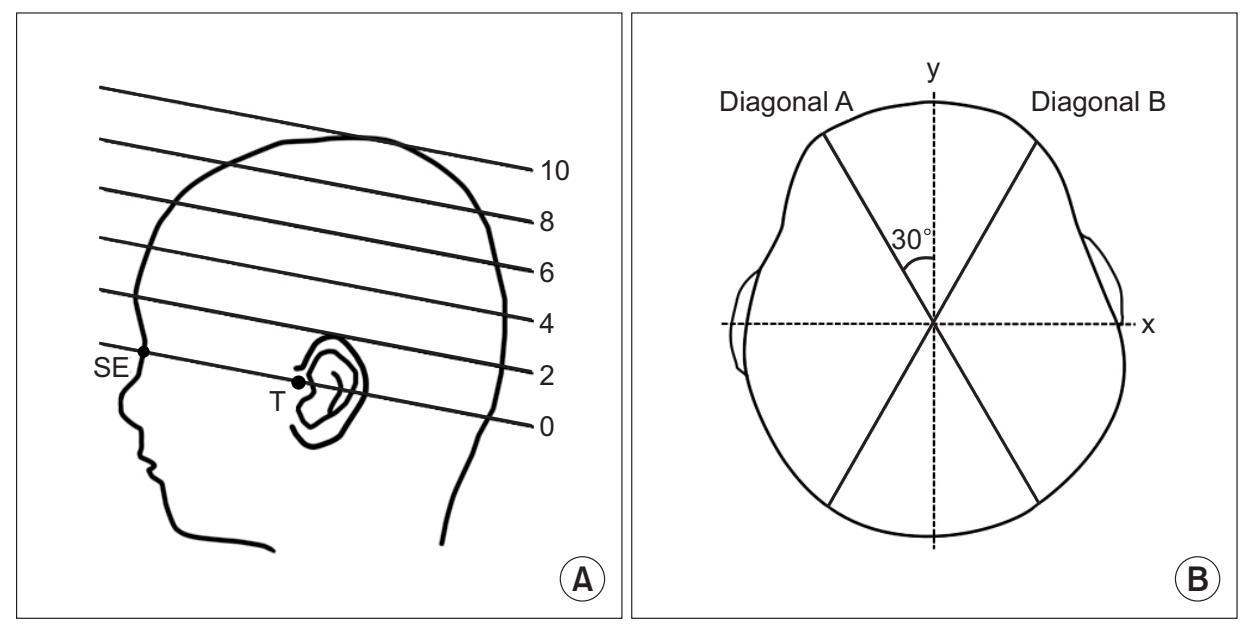

Fig. 1. (A) A cross-sectional plane through the sellion (SE) and both tragia (T) was set as the reference plane and designated the level 0 plane. The plane parallel to the level 0 plane that passed through the vertex of the head was designated as the level 10 plane. The portion of the cranium superior to the reference/level 0 plane was divided into 9 equally spaced cross-sectional planes, each parallel to the reference plane. (B) Cranial Vault Asymmetry Index $(\%)=$

long cranial diagonal $(\mathrm{mm})$-short cranial diagonal $(\mathrm{mm}) \times 100$. short cranial diagonal ( $\mathrm{mm})$ 
because of growth. In our study, a CVAI of less than 3.5\% was considered an objective treatment goal, since this value was defined in a previous study as a cut-off value that represented a significant asymmetry of the skull [14]. After the initial cranial-molding orthosis fitting, followup laser scans and clinic visits were done every 2 months. In follow-up visits, any newly developed adverse events and the duration of cranial-molding orthosis use per day were checked.

Parents' perspectives on the effectiveness of cranialmolding orthoses

Parent satisfaction was evaluated with the Goal Attainment Scale (GAS), which scores the extent to which a patient's individual goals are achieved during the course of the intervention [16]. GAS was first introduced in the 1960s by Kiresuk and Sherman [17] for assessing outcomes in mental-health settings. Since then, it has been modified and applied to many other areas including rehabilitation [18]. In this study, the parents' goal was defined by the parents as the degree of correction of the infant's skull asymmetry. The GAS score was assessed by how much the parents' goal was achieved after the treatment. The following scoring metric was used: 2 (much greater than expected outcome), 1 (greater than expected outcome), 0 (expected outcome after intervention), -1 (less than expected outcome), and -2 (much less than expected outcome).

Goal achievement ratings were converted to a GAS Tscore with a mean of 50 and standard deviation of 10 using the GAS calculation [16].

\section{Statistics}

Statistical analysis was performed using SPSS version 20.0 (IBM Corp., Chicago, IL, USA). Pearson correlation coefficient was used to calculate the relationship of the $\mathrm{T}$-score to the linearly related variables. Spearman rank correlation coefficient was used to measure the variables on an ordinal scale. An independent t-test was used to analyze the mean T-scores between the nominal scales groups. A chi-square $\left(\chi^{2}\right)$ statistic was used to investigate the differences in categorical variables. Cut-off points were analyzed by using a receiver operating characteristic (ROC) curve with a 95\% confidence interval (CI).

\section{RESULTS}

\section{Patient characteristics}

The mean subject age at the initiation of cranialmolding orthotic therapy was $24.3 \pm 8.4$ weeks. This age information was obtained with the corrected age. The corrected age of the youngest subject was 8.1 weeks, and the subject's chronological age was 17 weeks. The subject was able to hold up his head in sitting position at the beginning of the helmet therapy and his head circumference was within the normal range. The mean duration of cranial-molding orthotic therapy was $157.1 \pm 54.8$ days. The mean duration of cranial-molding orthosis usage per day was 21.2 \pm 2.7 hours (Table 1 ).

\section{Compliance with cranial-molding orthoses and adverse events}

The compliance with cranial-molding orthoses usage was $90.2 \%$ (74 of 82 infants). Eight infants discontinued wearing the cranial-molding orthosis because of the following: discomfort from wearing a cranial-molding orthosis $(n=4)$; otitis media which was unrelated to cranialmolding orthotic treatment $(n=1)$; heat rash $(n=1)$; and

Table 1. Basic characteristics of subjects $(n=74)$

\begin{tabular}{|c|c|}
\hline Characteristic & Value \\
\hline \multicolumn{2}{|l|}{ Sex } \\
\hline Male & 44 \\
\hline Female & 30 \\
\hline \multicolumn{2}{|l|}{ Type of birth } \\
\hline Fullterm & 65 \\
\hline Preterm & 9 \\
\hline NICU care history & $4(5.4)$ \\
\hline Age at initiation of therapy (wk) & $24.3 \pm 8.4(8.1-57.7)$ \\
\hline $\begin{array}{l}\text { Age at termination of therapy } \\
\text { (wk) }\end{array}$ & $46.8 \pm 13.4(22.3-77.9)$ \\
\hline Duration of therapy (day) & $157.1 \pm 54.8(63-297)$ \\
\hline $\begin{array}{l}\text { Hour of putting on cranial- } \\
\text { molding orthoses per day }\end{array}$ & $21.2 \pm 2.7(10-24)$ \\
\hline \multicolumn{2}{|l|}{ CVAI (\%) at initiation } \\
\hline Level 3 & $8.4 \pm 3.2(3.5-19.6)$ \\
\hline Level 5 & $8.6 \pm 3.3(1.9-18.0)$ \\
\hline
\end{tabular}

Values are presented as number (\%) or mean \pm standard deviation (min-max).

NICU, neonatal intensive care unit; CVAI, Cranial Vault Asymmetry Index. 
cause unknown $(n=2)$. These 8 infants were excluded from this study because we could not obtain information about the evaluation by their parents or the anthropometric outcomes.

The adverse events associated with cranial-molding orthotic treatment are presented in Table 2 . The adverse events are also presented for infants who discontinued treatment. During the study, 53 out of 82 infants (65\%) developed adverse events from the cranial-molding orthoses. Heat rash was observed in 29 cases (35.4\%) and was the most common adverse event. One infant ceased cranial-molding orthotic treatment because of heat rash. Pressure sores were observed in 21 cases (25.6\%). Other side effects included itchiness (7.3\%), discomfort (4.9\%), bacterial abscess $(1.2 \%)$, and corneous (1.2\%). Eight of the infants $(10.8 \%)$ had two or more adverse events. Most adverse events were mild and not complicated. All of the pressure sores presented as round, red-colored marks

Table 2. Adverse events of cranial-molding orthoses therapy $(\mathrm{n}=82)$

\begin{tabular}{lc}
\hline \multicolumn{1}{c}{ Adverse events } & Value \\
\hline Heat rash & $29(35.4)$ \\
Pressure sore & $21(25.6)$ \\
Itching sense & $6(7.3)$ \\
Failure to putting on due to uncomfortable & $4(4.9)$ \\
Bacterial abscess & $1(1.2)$ \\
Corneous & $1(1.2)$ \\
Two or more adverse events & $8(9.8)$ \\
None & $29(35.4)$ \\
\hline
\end{tabular}

Values are presented as number (\%).

Infants who stopped the cranial-molding orthoses therapy were involved. and did not damage the dermis.

\section{Effectiveness of cranial-molding orthoses}

The mean terminal CVAI at levels 3 and 5 were $3.5 \pm 1.8$ and 3.6 \pm 2.0 , respectively. The mean difference of CVAI between initiation and termination at levels 3 and 5 was $4.9 \pm 2.4$ and 4.9 \pm 2.6 , respectively. On the goal attainment scale, a score of 0 (expected outcome after intervention) was the most common answer for parents. A score of 0 was reported in 24 (32.4\%) of the parents' answers. Scores of 1 and of -1 were each reported in $20(27.0 \%)$ of the parents' answers. A score of 2 was reported in 9 (12.2\%) of the parents' answers, and a score of -2 was reported in $1(1.4 \%)$ of the parents' answers. The mean GAS T-score was $51.9 \pm 10.2$. A GAS T-score of 0 or more was reported for $71.6 \%$ of parents, which means that the goals of cranial-molding orthotic treatment were achieved in more than $70 \%$ of cases. The GAS T-score was significantly related to age $(p<0.001)$ (Fig. 2$)$. The overall duration of therapy and the duration of orthosis usage per day were not related to the GAS T-score. At levels 3 and 5, the initial CVAI and the differences between CVAI values over the course of treatment $(\mathrm{p}<0.001)$ were significantly related to the GAS T-score (Fig. 3). There was no association between the terminal CVAI and the GAS T-score. In order to find out whether there was a relationship between adverse events and the GAS T-score, the means of the GAS T-scores between the group with adverse events and the group without adverse events were compared. Although the mean of the GAS T-score in the group without adverse events was higher than the mean of the GAS T-score in the group with adverse events (53.9 vs. 51.1, respectively), the difference was not significant ( $\mathrm{p}=0.498)$. Last, the number and the types of adverse events were not related
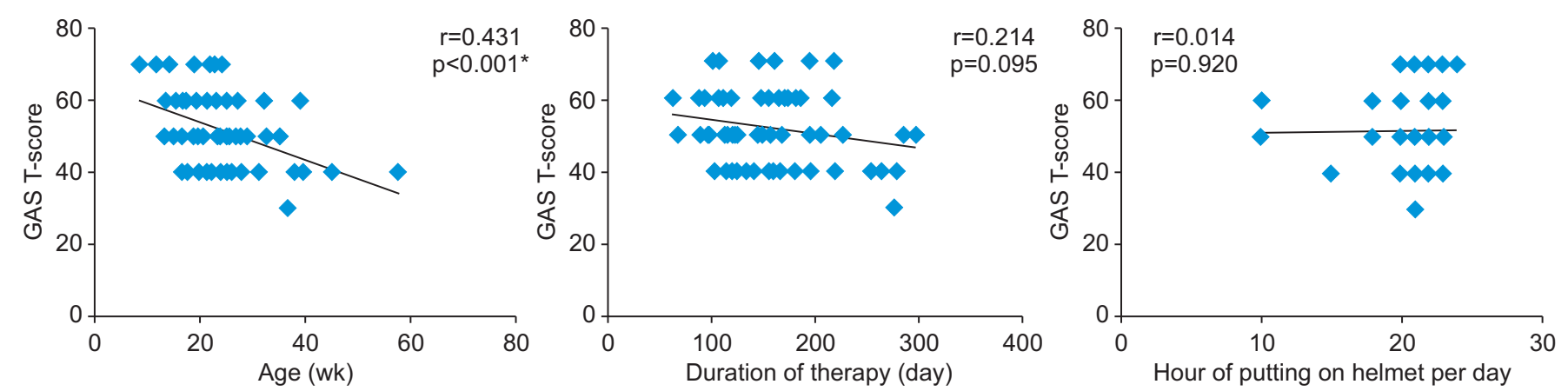

Fig. 2. Age, duration of therapy, and hours of putting on cranial-molding orthoses versus GAS T-score. The GAS Tscore was significantly related to the age. GAS, Goal Attainment Scale. 

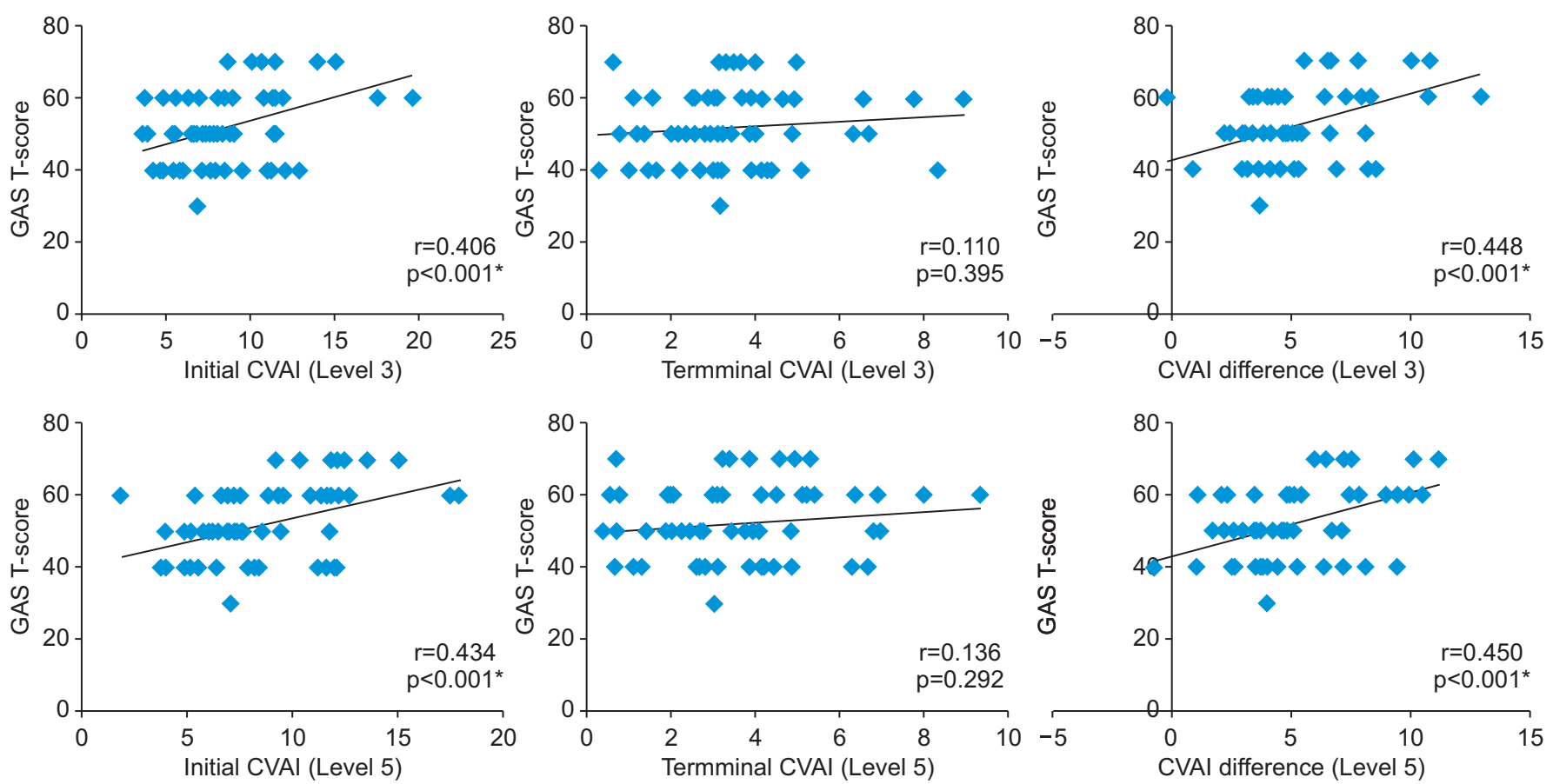

Fig. 3. Initial CVAI (left), terminal CVAI (center), difference of CVAI (right) from initiation and to termination versus GAS T-score. The initial CVAI and the difference of CVAI from the initiation to the termination of the treatment at level 3 and level 5 were significantly related to the GAS T-score. CVAI, Cranial Vault Asymmetry Index; GAS, Goal Attainment Scale.

to the GAS T-score (Table 3).

The difference in the CVAI at level 3, from the initiation to the termination of the treatment, was significantly related to age $(\mathrm{p}<0.003)$ and to the CVAI at treatment initiation $(\mathrm{p}<0.001)$ (Fig. 4). The subject's gender, the overall duration of cranial-molding orthotic therapy, and the duration of orthotic therapy per day were not related to the difference in the CVAI at level 3 over the course of treatment. The difference in the CVAI at level 5, from the initiation to the termination of the treatment, was significantly related to age $(\mathrm{p}=0.003)$ and to the CVAI at treatment initiation $(\mathrm{p}<0.001)$ (Fig. 4). The subject's gender, the overall duration of cranial-molding orthotic therapy, and the duration of cranial-molding orthotic therapy per day were not related to the difference of CVAI at level 5 over the course of treatment.

\section{ROC curve analysis}

The cut-off points that indicated high parental satisfaction were analyzed by using a ROC curve with a $95 \%$ CI in order to survey the statistical influence of various parameters, which included the subject age at the start of therapy, the initial CVAI, and the difference in the CVAI
Table 3. Outcomes of cranial-molding orthoses therapy $(\mathrm{n}=74)$

\begin{tabular}{|cc|}
\hline \multicolumn{1}{c}{ Measurement } & Value \\
\hline CVAI at termination (\%) & $3.5 \pm 1.8(0.20-9.0)$ \\
\hline Level 3 & $3.6 \pm 2.0(0.1-9.4)$ \\
\hline Level 5 & \\
$\begin{array}{l}\text { Difference of CVAI from } \\
\text { initiation to termination (\%) }\end{array}$ & \\
\hline Level 3 & $4.9 \pm 2.4(-0.2-12.9)$ \\
\hline Level 5 & $4.9 \pm 2.6(-0.8-11.2)$ \\
\hline GAS & $1(1.4)$ \\
\hline-2 (much worse) & $20(27.0)$ \\
\hline-1 (somewhat worse) & $24(32.4)$ \\
\hline 0 (achieved) & $20(27.0)$ \\
\hline 1 (somewhat better) & $9(12.2)$ \\
\hline 2 (much better) & $51.9 \pm 10.2(30-70)$ \\
\hline GAS T-score
\end{tabular}

Values are presented as mean \pm standard deviation (minmax) or number (\%).

Level 3 and level 5 are cross-sectional planes, each parallel to the level 0 plane (Fig. 1A).

CVAI, Cranial Vault Asymmetry Index; GAS, Goal Attainment Scale. 

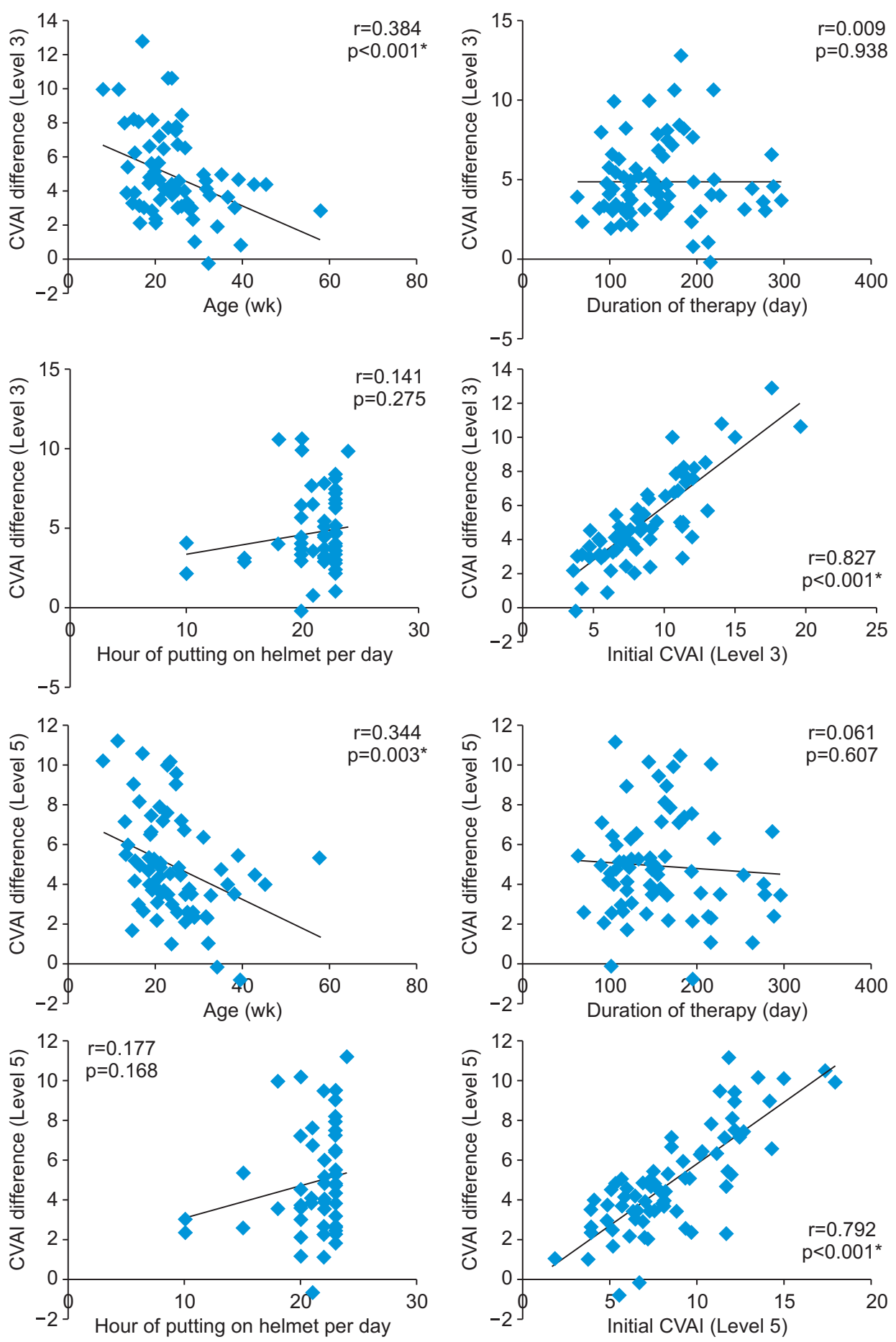

Fig. 4. Age, duration of therapy, hours of putting on cranial-molding orthoses per day and initial Cranial Vault Asymmetry Index (CVAI) versus difference of CVAI from initiation and to termination (level 3). The difference of CVAI at level 3 and level 5 from the initiation to the termination of the treatment were significantly related to the age and the initial CVAI.

over the course of treatment. Here, we defined high parental satisfaction as a GAS T-score equal to or more than 50 . When the treatment was initiated at age 24.5 weeks, the area under the ROC curve (AUC) was 0.282 (CI, 0.1440.421 ), which indicates that infants who had started cranial-molding orthotic treatment younger than 24.5 weeks showed higher parental satisfaction $(p=0.007)$. When the cranial-molding orthoses were applied to infants who had an initial CVAI of more than $8.50 \%$, the AUC was 0.631 (CI, 0.471-0.790), which indicated that infants with an initial CVAI of more than $8.50 \%$ were more likely to achieve higher parental satisfaction $(p>0.05)$. When the difference of CVAI over the course treatment was more than 4.50, the AUC was 0.619 (CI, 0.468-0.770). This indi- 
cates that the difference of CVAI during the treatment was more than 4.50 and was more likely to achieve a higher parent satisfaction ( $\mathrm{p}>0.05)$.

In order to analyze the statistical influence of different parameters, including the subject age and CVAI at the start of therapy, cut-off points that indicated a successful therapy were analyzed by using a ROC curve with a $95 \%$ CI. We defined successful therapy as a scenario where the CVAI at a severe level of asymmetry decreased to less than $3.5 \%$ after the treatment. When the treatment was initiated at age 23.5 weeks, the AUC was 0.31 (CI, 0.190$0.433)$, and when the cranial-molding orthoses were applied to infants who had an initial CVAI of less than $8.50 \%$, the AUC was 0.184 (CI, 0.087-0.281). This indicates that infants who had started cranial-molding orthotic treatment younger than 23.5 weeks and had an initial CVAI of less than $8.50 \%$ showed higher rates of therapeutic success ( $\mathrm{p}=0.005$ and $\mathrm{p}=0.000$, respectively).

\section{DISCUSSION}

In this study, the GAS T-score was significantly related to infant age, initial CVAI, and the difference in the CVAI over the course of treatment. Parents perceived that cranial-molding orthoses were more effective when their children had severe initial skull asymmetry and when the orthoses were applied earlier in infancy. Furthermore, greater asymmetry correction during the treatment was related to parents' perception of goal achievement. On the other hand, the GAS T-score was not related to the terminal CVAI. Therefore, parental satisfaction was affected by how much asymmetry was corrected through the treatment, and not by how much asymmetry existed after the treatment. The GAS T-score was not related to the overall therapy duration and the duration of cranialmolding orthosis usage per day, perhaps because of observing only minimal differences in overall therapy duration and duration of cranial-molding orthosis usage per day between infants.

The mean GAS T-score for cranial-molding orthoses in this study was 51.94. Elwood et al. [12] reported that parents rated head-shape improvement a 4.06 out of 5 when the cranial-molding orthotic treatment was applied. It is inappropriate to compare the results of this current study to the results of the Elwood et al. [12], since different evaluation tools were used in the studies. However, we did observe a GAS T-score of 0 or more in more than $70 \%$ of parents, which was comparable to the results of the Elwood et al. [12]. Parents' perspectives are affected by many factors that include not only the degree of asymmetry correction, but also the cost, the difficulty of the treatment, the concern over adverse events, and the propensity of an individual parent. Understanding the parents' perspectives is considered important in decision making and in the evaluation of interventions.

There were 48 ( $65 \%$ of the subjects) who had adverse events associated with the cranial-molding orthoses during the study. According to a previous study detailing the adverse events associated with the orthoses, the rate of adverse events was approximately $25 \%$. In this study, pressure sores were the most common adverse events of cranial-molding orthoses (10.9\%), followed by ethanol erythema (7.4\%), improper fit (4.4\%), and skin infection (1.3\%) [19]. A subsequent study showed that 54 children (26.3\%) had minor adverse events during the course of cranial-molding orthotic treatment, which included pressure sores $(13.7 \%)$, ethanol erythema $(2.9 \%)$, skin erosion/skin infection (4.3\%), or improper fit (5.4\%) [20]. A large prospective study of 260 patients who were treated with custom cranial-molding orthoses reported a morbidity rate of $0 \%$ [21]. Another prospective study reported that all infants had one or more adverse events related to cranial-molding orthoses: problems with acceptance of the helmet (24\%), skin irritation (96\%), increased sweating $(71 \%)$, unpleasant odor of the helmet $(76 \%)$, pain associated with the helmet (33\%), and parents' feeling hindered from cuddling their child (77\%) [10].

However, only 35 of the parents were surveyed for adverse events during the cranial-molding orthoses, and the severity of those adverse events was not discussed in this study.

An explanation for the high rate of the adverse events in this study may be that it included very mild heat rash as an adverse event. However, most of the adverse events were mild and not complicated. All of the pressure sores presented as round, red-colored marks and did not damage the dermis. This study suggests that cranial-molding orthotic treatment is a relatively safe treatment. Although there were no serious side effects, the rate of adverse events was as high as $65 \%$; hence medical monitoring should be performed during cranial-molding orthotic treatment. 
The difference in the CVAI between the initiation and the termination of the treatment was significantly related to the infant age and the initial CVAI, as is consistent with previous studies $[22,23]$. When cut-off points were analyzed by using an ROC curve with a $95 \%$ CI, the infants who had started cranial-molding orthotic treatment younger than 23.5 weeks and had an initial CVAI of less than $8.50 \%$ showed a higher rate of therapeutic success ( $\mathrm{p}=0.005$ and $\mathrm{p}=0.000)$. Freudlsperger et al. [24] reported a statistically relevant difference in the rate of successful treatment for a CVAI of $9.5 \%(\mathrm{p}<0.001)$ and an initial treatment age of 25.5 weeks $(p=0.0345)$. Studies by Graham et al. [25] and Seruya et al. [26] concluded that early cranial-molding orthotic treatment before 8 months resulted in better outcomes than did late treatment after 8 months and that infants treated with cranial-molding orthoses when they were older required a longer treatment. Seruya et al. [26] also reported that improvement was still seen even in infants over 12 months of age at the time of cranial-molding orthotic treatment initiation.

Several limitations exist in this study. Even though a growing body of evidence supports the sensitivity of GAS over other standard measures, it was not easy to define specific goals for the correction of deformities. Therefore, we had to ask parents if their goals, which they set before treatment, were met after the cranial-molding orthotic treatment. Furthermore, 8 infants were excluded in the final analysis because they had ceased wearing the orthosis during the study period. Therefore, the achievement of cranial-molding orthotic treatment goals in this study may be overestimated.

In this study, the mean subject age at the initiation of cranial-molding orthotic therapy was $24.3 \pm 8.4$ weeks and 50 infants initiated the therapy at the age of 6 months or less. Relatively young infants were included in this study to examine the parents' perspectives and clinical effect of cranial orthotic therapy in young age. There were 26 infants (35\%) who had mild asymmetric severity, defined as CVAI less than 7\%, who were enrolled in this study to assess how the effect of cranial-molding orthotic therapy differs according to the severity [15]. According to the results of this study, parent's satisfaction and therapeutic efficacy were greater when cranial-molding orthoses were applied earlier in infancy. In addition, the ratio of normal CVAI values after cranial-molding orthotic treatment was higher in infants with an initially mild asym- metric severity. Further studies are needed to establish guidelines for appropriate age and severity of cranialmolding orthotic therapy.

Family-centered treatment is considered to be a best practice in early intervention and pediatric rehabilitation [27]. There has been an increasing understanding of the family's role in a child's life and the importance of parental insights into their child's abilities and needs. Therefore, outcomes should include family-centered assessment. Much of the research on quality of care has focused on the key outcomes of parental satisfaction, on reduced stress and worry, and on adherence to therapy programs, and these parental outcomes should certainly be considered [28]. However, there is a lack of research on standard assessment of parents' perspectives on cranial-molding orthotic treatment. This study used a family-centered assessment in cranial-molding orthotic treatment and demonstrated some factors that related to the parents' perspectives. Therefore, cranial-molding orthotic treatment in plagiocephaly can be more individualized and child-centered and/or family-centered based on the results of this study.

Cranial-molding orthotic treatment is becoming more popular in Korea despite a lack of strong evidence. Anthropometric parameters including the CVAI cannot describe the whole nature of the plagiocephaly. It is necessary to create a new tool to evaluate parents' perspectives on cranial-molding orthoses in a more objective way. Also, adverse events, including developmental delays, should be monitored and documented in cranial-molding orthotic treatment. Therefore, results of this study will contribute to developing methodology in the study of plagiocephaly for evidence-based practice.

In conclusion, although the rate of adverse events for cranial-molding orthotic treatment is relatively high, the anthropometric improvement, the treatment compliance, and the degree of parents' perspectives are high. However, it is necessary to perform medical monitoring during cranial-molding orthotic treatment and to develop a new cranial-molding orthosis that can lower the rate of adverse events in the future. Finally, this study provides basic information for conducting a patient-centered assessment in infants and parents as an important criterion for establishing cranial-molding orthotic treatment as an evidence-based practice. 


\section{CONFLICT OF INTEREST}

No potential conflict of interest relevant to this article was reported.

\section{ACKNOWLEDGMENTS}

This work was supported by the ICT R\&D Program of IITP/MSIP (No. B0101-17-1081, Development of ICTbased software platforms and service technologies for medical 3D-printing applications).

\section{REFERENCES}

1. Ellenbogen RG, Gruss JS, Cunningham ML. Update on craniofacial surgery: the differential diagnosis of lambdoid synostosis/posterior plagiocephaly. Clin Neurosurg 2000;47:303-18.

2. Mortenson P, Steinbok P, Smith D. Deformational plagiocephaly and orthotic treatment: indications and limitations. Childs Nerv Syst 2012;28:1407-12.

3. Looman WS, Flannery AB. Evidence-based care of the child with deformational plagiocephaly, Part I: assessment and diagnosis. J Pediatr Health Care 2012;26:242-50.

4. Hutchison BL, Hutchison LA, Thompson JM, Mitchell EA. Plagiocephaly and brachycephaly in the first two years of life: a prospective cohort study. Pediatrics 2004;114:970-80.

5. Collett B, Breiger D, King D, Cunningham M, Speltz M. Neurodevelopmental implications of "deformational" plagiocephaly. J Dev Behav Pediatr 2005;26:379-89.

6. Boere-Boonekamp MM, van der Linden-Kuiper LT. Positional preference: prevalence in infants and follow-up after two years. Pediatrics 2001;107:339-43.

7. Robinson S, Proctor M. Diagnosis and management of deformational plagiocephaly. J Neurosurg Pediatr 2009;3:284-95.

8. Tamber MS, Nikas D, Beier A, Baird LC, Bauer DF, Durham S, et al. Congress of neurological surgeons systematic review and evidence-based guideline on the role of cranial molding orthosis (Helmet) therapy for patients with positional plagiocephaly. Neurosurgery 2016;79:E632-E633.

9. van Wijk RM, van Vlimmeren LA, Groothuis-Oudshoorn CG, Van der Ploeg CP, Ijzerman MJ, Boere-
Boonekamp MM. Helmet therapy in infants with positional skull deformation: randomised controlled trial. BMJ 2014;348:g2741.

10. Mulliken JB, Vander Woude DL, Hansen M, LaBrie RA, Scott RM. Analysis of posterior plagiocephaly: deformational versus synostotic. Plast Reconstr Surg 1999;103:371-80.

11. Stadnick NA, Drahota A, Brookman-Frazee L. Parent perspectives of an evidence-based intervention for children with autism served in community mental health clinics. J Child Fam Stud 2013;22:414-22.

12. Elwood ET, Petronio J, Wood RJ. Parental satisfaction with the CranioCap: a new cranial orthosis for deformational plagiocephaly. Cleft Palate Craniofac J 2005;42:340-3.

13. Katzel EB, Koltz PF, Sbitany H, Girotto JA. Treatment of plagiocephaly with helmet molding therapy: do actual results mimic perception? Cleft Palate Craniofac J 2011;48:205-9.

14. Loveday BP, de Chalain TB. Active counterpositioning or orthotic device to treat positional plagiocephaly? J Craniofac Surg 2001;12:308-13.

15. Mortenson PA, Steinbok P. Quantifying positional plagiocephaly: reliability and validity of anthropometric measurements. J Craniofac Surg 2006;17:413-9.

16. Turner-Stokes L. Goal attainment scaling (GAS) in rehabilitation: a practical guide. Clin Rehabil 2009;23:362-70.

17. Kiresuk TJ, Sherman RE. Goal attainment scaling: a general method for evaluating comprehensive community mental health programs. Community Ment Health J 1968;4:443-53.

18. Rockwood K, Joyce B, Stolee P. Use of goal attainment scaling in measuring clinically important change in cognitive rehabilitation patients. J Clin Epidemiol 1997;50:581-8.

19. Wilbrand JF, Wilbrand M, Malik CY, Howaldt HP, Streckbein $\mathrm{P}$, Schaaf $\mathrm{H}$, et al. Complications in helmet therapy. J Craniomaxillofac Surg 2012;40:341-6.

20. Freudlsperger C, Bodem JP, Kargus S, CastrillonOberndorfer G, Hoffman J, Engel M. The incidence of complications associated with molding helmet therapy: an avoidable risk in the treatment of positional head deformities? J Craniofac Surg 2015;26:e299-302.

21. de Ribaupierre S, Vernet O, Rilliet B, Cavin B, Kalina D, Leyvraz P. Posterior positional plagiocephaly treated 
with cranial remodeling orthosis. Swiss Med Wkly 2007;137:368-72.

22. Vles JS, Colla C, Weber JW, Beuls E, Wilmink J, Kingma $\mathrm{H}$. Helmet versus nonhelmet treatment in nonsynostotic positional posterior plagiocephaly. J Craniofac Surg 2000;11:572-4.

23. Kluba S, Kraut W, Calgeer B, Reinert S, Krimmel M. Treatment of positional plagiocephaly: helmet or no helmet? J Craniomaxillofac Surg. 2014;42:683-8.

24. Freudlsperger C, Steinmacher S, Saure D, Bodem JP, Kuhle R, Hoffmann J, et al. Impact of severity and therapy onset on helmet therapy in positional plagiocephaly. J Craniomaxillofac Surg 2016;44:110-5.

25. Graham JM Jr, Gomez M, Halberg A, Earl DL, Kreutzman JT, Cui J, et al. Management of deforma- tional plagiocephaly: repositioning versus orthotic therapy. J Pediatr 2005;146:258-62.

26. Seruya M, Oh AK, Taylor JH, Sauerhammer TM, Rogers GF. Helmet treatment of deformational plagiocephaly: the relationship between age at initiation and rate of correction. Plast Reconstr Surg 2013;131:55e61 e.

27. Bailey DB Jr, Buysse V, Edmondson R, Smith TM. Creating family-centered services in early intervention: perceptions of professionals in four states. Except Child 1992;58:298-309.

28. King G, King S, Rosenbaum P. Interpersonal aspects of care-giving and client outcomes: a review of the literature. Ambul Child Health 1996;2:151-60. 